\title{
DISTAL MINIMAL FLOW IS COALESCENT
}

\section{TA-SUN WU}

A transformation group $(X, T)$ is coalescent if every homomorphism of $(X, T)$ into itself is an isomorphism [1]. A transformation group $(X, T)$ is a distal minimal flow if $X$ is a compact metric space, $T$ a locally compact group, and $(X, T)$ is a distal minimal transformation group [2], [3]. It has been known that:

Theorem 1 [1, Theorem 7]. Every almost periodic transformation group is coalescent.

It has also been known that there exists minimal transformation groups which are not coalescent [1]. In fact, we have the examples of nonisomorphic minimal sets having homomorphisms from each onto the other. The details of these examples will appear elsewhere.

The proof we give here depends heavily on H. Furstenberg's structure theorem of distal flow [3]. We shall follow the definitions and notations in [3] strictly and shall not repeat them here. However, we shall make the following remark.

REMARK. It was proved in [3, pp. 481-482] that every coördinate bundle with structure group $M$ is an $M$-bundle extension over the base space. The converse is not true in general. Witness:

Let $G=\prod_{i=1}^{\infty} G_{i} ; G_{i}=$ circle group $=\{z:|z|=1\}, M \subseteq G ; M$ $=\prod_{i=1}^{\infty} M_{i}, M_{i}=\{1,-1\} \subseteq G_{i} . G$ has an invariant metric $\rho . G$ is an $M$-bundle extension over $G / M$ with respect to the function $\rho$. But it is known that $(G, G / M, M)$ is not a coördinate bundle. This. a question in $[3$, p. 482].

Now we proceed.

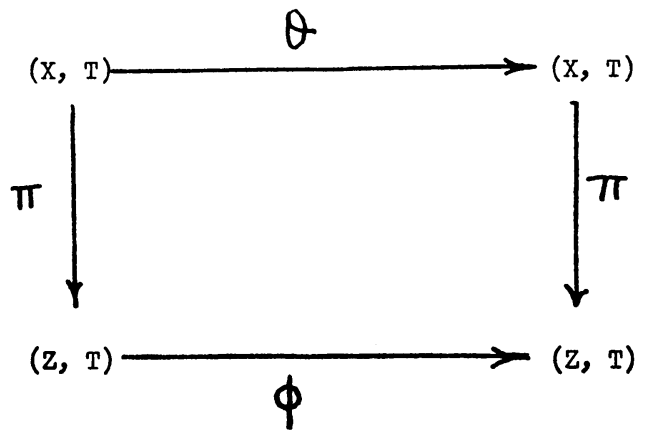

Received by the editors November 21, 1966. 
Lemma 1. Suppose $(X, T)$ is a distal minimal flow, and the preceding diagram commutes where $\theta, \Pi, \phi$ are homomorphisms.

Let $(Y, T),\left(Y^{\prime}, T\right)$ be the maximal isometric extensions (see $[3$, Proposition 13.1] for the definition and existence) with respect to $\Pi$ I, $\Pi \theta$ respectively. We shall write $\alpha, \alpha^{\prime}, \beta, \beta^{\prime}$ for the corresponding homomorphisms (cf. the following diagrams) in regard to the maximal isometric extensions. If $\phi$ is an isomorphism, then there exists an isomorphism $\Delta:(Y, T) \rightarrow(Y, T)$ such that the following diagram commutes.

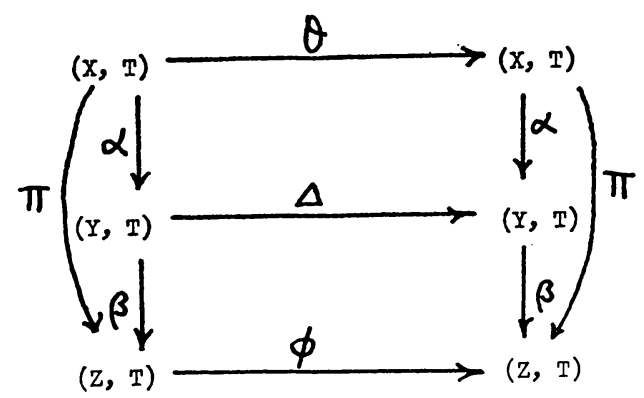

Proof. Observe the following diagram.

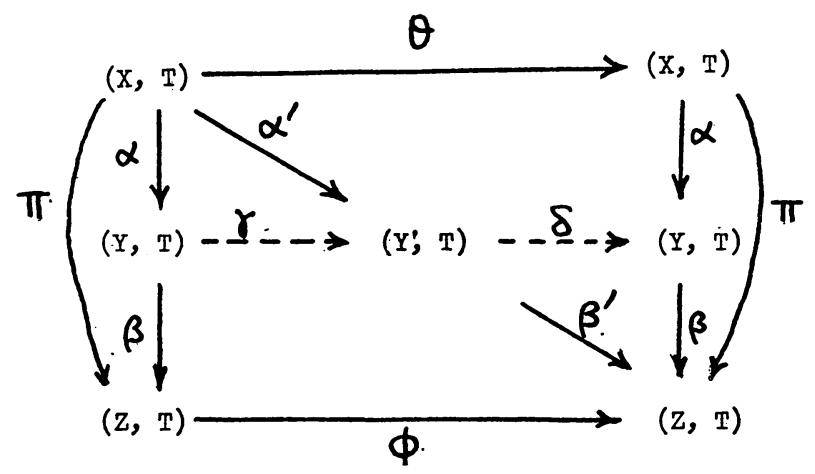

We note $\left(Y^{\prime}, T\right)$ is an isometric extension over $(Z, T)$ with respect to the homomorphism $\phi^{-1} \beta^{\prime}$. Since $\phi^{-1} \beta^{\prime}\left(Y^{\prime}, T\right)=\phi^{-1} \beta^{\prime} \alpha^{\prime}(X, T)=$ $\phi^{-1} \Pi \theta(X, T)=\phi^{-1} \phi \Pi(X, T)=\Pi(X, T)$, and $(Y, T)$ is a maximal isometric extension with respect to $\Pi$, there is a homomorphism $\gamma:(Y, T)$ $\rightarrow\left(Y^{\prime}, T\right)$. As $\beta \alpha \theta(X, T)=\beta(Y, T)=\Pi \theta(X, T), \beta:(Y, T) \rightarrow(Z, T)$ is an isometric extension of $(Z, T)$ with respect to $\Pi \theta$. There is a homomorphism $\delta:\left(Y^{\prime}, T\right) \rightarrow(Y, T)$. Let $\Delta=\delta \gamma:(Y, T) \rightarrow(Y, T)$. For $z \in Z$ we denote the fiber over $z$ under $\beta$ by $Y_{z}=\{y: y \in Y, \beta(y)=z\}$, then $\Delta=\delta \gamma: Y_{z} \rightarrow Y_{\phi(z)}$. Denote the enveloping semigroup of $(Y, T)$ by $G$, 
and the subgroup of $G$ which leaves $Y_{z}$ invariant by $G_{z}[3$, Section 3] (the enveloping semigroup is called Ellis' group in [3]), then $\left(Y_{z}, G_{z}\right)$ is an almost periodic minimal flow. If $y \in Y_{z}, y^{\prime} \in Y_{z}^{\prime}$, then there is an element $f \in G$ such that $f(z)=z^{\prime}$ since $G$ is transitive on $Y$. Define the transformation group $\left(Y_{z}^{\prime}, G_{z}\right)$ by $\eta: G_{z} \times Y_{z}^{\prime} \rightarrow Y_{z}^{\prime}, \eta(g, b)=f g f^{-1}(b)$, then $\left(Y_{z}^{\prime}, G_{z}\right)$ is an almost periodic minimal set since the actions are isometrics (cf. definition of isometric extension ${ }^{1}$ ). Observe the map $\phi_{f}:\left(Y_{z}, G_{z}\right) \rightarrow\left(Y_{z}^{\prime}, G_{z}\right), \phi_{f}(a)=f(a)$ is an isomorphism. By Theorem 1, $\Delta \mid Y_{z}: Y_{z} \rightarrow Y_{\phi(z)}$ has to be an isomorphism for each $z \in Z$, a fortiori, $\Delta$ is an isomorphism.

THEOREM 2. Every distal minimal flow $(X, T)$ is coalescent.

Proof. By the results in [3], $(X, T)$ is the limit of a normal quasiisometric system of $\left\{\left(X_{\xi}, T\right), \xi \leqq \eta\right\}$ where (1) $\eta$ is an ordinal number, and (2) $\left(X_{\xi}, T\right)=\Pi_{\xi}(X, T)$ is a subflow of $(X, T),(3)\left(X_{\xi+1}, T\right)$ is maximal isometric extension of $\left(X_{\xi}, T\right)=\Pi_{\xi+1, \xi}\left(X_{\xi+1}, T\right)$, and (4) $\left(X_{0}, T\right)$ is a trivial flow, i.e. $X_{0}$ is a singleton. If $\theta$ is an endomorphism of $(X, T), \theta$ induces the (trivial) automorphism $\Delta_{0}$ of $\left(X_{0}, T\right)$. By Lemma $1, \theta$ induces an automorphism $\Delta$, of $\left(X_{1}, T\right)$. Now, we shall prove the theorem by transfinite induction. If $\xi$ is not a limit ordinal, we use Lemma 1 , to show the existence of automorphism $\Delta_{\xi+1}$ of $\left(X_{\xi+1}, T\right)$. If $\xi$ is a limit ordinal then $\left(X_{\xi}, T\right)$ is the limit of $\left\{\left(X_{\zeta}, T\right): \zeta<\xi\right\}$. Consider the following diagram:

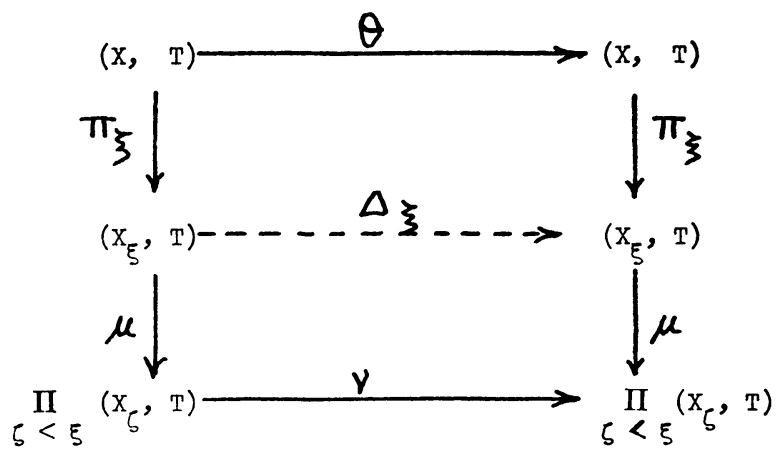

where $\mu(x)_{\zeta}=\prod_{\xi, \zeta}(x)$, for $x \in X_{\xi} ; \nu(y)_{\zeta}=\Delta_{\zeta}\left(y_{\zeta}\right)$ for $y \in \prod_{\zeta<\xi} X_{\zeta}$. Since each $\Delta_{\zeta}$ is an isomorphism, so is $\nu$. By definition, ([3, Definition 2.3]) $\mu$ is an isomorphism. Hence $\mu^{-1} \nu \mu$ induces an isomorphism $\Delta_{\zeta}$. It is not hard to see that the diagram commutes. Thus the proof is complete.

1 We note here the restriction of elements of $G$ on each fiber are isometrics. It is known for $g \in G$, in general $g$ is not continuous on $Y$ if $(Y, T)$ is not almost periodic. 
Added in Proof (January 15, 1968). The proof of Lemma 1 has a gap (last three lines). Thus following present proof, the Theorem 2 should be modified to "Each endomorphism of $(X, T)$ can be factored through the normal extensions."

\section{REFERENCES}

1. Joseph Auslander, Endomorphism of minimal sets, Duke Math J. 30 (1963), 605-614.

2. R. Ellis, Distal transformation group, Pacific J. Math. 8 (1958), 401-405.

3. H. Furstenberg, The structure of distal flows, Amer. J. Math. 85 (1963), 477-515.

Case Institute of Technology 